\title{
Effective Coping Strategies Employed by University Students in Three Sensitivity Groups:
}

\section{A Quantitative Text Analysis}

Kosuke Yano ${ }^{\mathrm{a}, b^{*}}$, Shintaro Endo ${ }^{\mathrm{c}}$, Shunsuke Kimura ${ }^{\mathrm{d}}$, and Kazuo Oishi ${ }^{\mathrm{d}}$

\author{
${ }^{a}$ Graduate School of Community and Human Services, Rikkyo University \\ ${ }^{b}$ Research Fellow of Japan Society for the Promotion of Science (DC1) \\ ${ }^{\mathrm{c}}$ Research Center for Youth Education, National Institution for Youth Education \\ ${ }^{\mathrm{d}}$ College of Community and Human Services, Rikkyo University \\ *Corresponding author \\ Graduate School of Community and Human Services, Rikkyo University \\ 1-2-26 Kitano, Niiza-shi, Saitama, JAPAN \\ E-mail: kosuke.yano1012@gmail.com
}

\section{Acknowledgements}

The authors would like to thank Editage (www.editage.jp) for English-language editing and translation services.

\section{Conflicts of Interest}

There is no conflict of interest with regard to this manuscript.

\section{Funding}

This work was supported by JSPS KAKENHI Grant number JP18K03079, JP19J20902.

\section{Ethical Approval}

This study was approved by the ethics review board of College of Community and Human Services in Rikkyo University (No. KOMI19014A).

\section{Data Availability Statement}

Due to the nature of this research, participants of this study did not agree for their data to be shared publicly, so supporting data is not available. 
Effective Coping Strategies Employed by University Students in Three Sensitivity Groups:

A Quantitative Text Analysis

\begin{abstract}
People differ in their sensitivity to internal and external stimuli, falling into one of three sensitivity groups (low, medium, and high). Studies have pointed out that individual differences in sensitivity should be considered in psychological intervention settings. This study aimed to explore effective coping strategies in the three sensitivity groups. In total, 692 Japanese university students (389 females; $M_{\text {age }}=20.6 \pm 1.4$ years) responded to an open-ended question about the coping strategies they employ, and to two self-report measures assessing their level of sensitivity and mental health. A series of co-occurrence network analyses with two grouping variables (i.e., better or poorer mental health) suggested that effective coping strategies differed among the three sensitivity groups.
\end{abstract}

Keywords: sensory processing sensitivity; coping; quantitative methods; students; temperament 
Effective Coping Strategies Employed by University Students in Three Sensitivity Groups: A Quantitative Text Analysis

\section{Introduction}

\section{Mental Health Problems in University Students and Interventions for Improving Coping Skills}

University students experience various stressors such as psychological conflict derived from developmental issues (i.e., establishing identity), changes in their lifestyles and interpersonal relationships, and academic stress (American College Health Association, 2009). Consequently, they tend to have more mental health problems than the general population (e.g., Auerbach et al., 2016; Nippon Foundation Suicide Prevention Project, 2019). Although poorer mental health is a risk factor for poorer academic performance (Andrews \& Wilding, 2004), dropping out of school (Pascoe et al., 2020), and/or suicidal thoughts and attempts (Mortier et al., 2018), only 16.4\% of university students with mental health problems seek professional support (Auerbach et al., 2016). Therefore, universities have conducted universally designed interventions to prevent their students from experiencing such problems. Because university students are exposed to stressors in their daily lives (Acharya et al., 2018), most approaches conducted in universities aim to enhance their skills to cope with these stressors and/or their related negative emotions (Amanvermez et al., 2020).

However, there are individual differences in the efficiency of universally designed approaches for better mental health (Werner-Seidler et al., 2017). In addition, a recent meta-analysis reported that interventions in educational institutions, including universities, demonstrated small-to-moderate effect sizes (Amanvermez et al., 2020; Werner-Seidler et al., 2017), suggesting that more effective intervention programs are needed to ensure more benefits for all participants. Therefore, this study aimed to provide evidence for creating an intervention program that aims to improve students' coping skills. 
A great many studies on coping have been conducted and have provided a framework classifying coping strategies into such categories as problem-focused and emotion-focused coping (Lazarus \& Folkman, 1984) ${ }^{1}$ or primary control and secondary control coping (Rothbaum et al., 1982). ${ }^{2}$ In general, it has been suggested that the strategies of problem-focused or primary control coping are associated with better mental health and less frequent maladaptive behavior, whereas emotion-focused or secondary control coping are associated with physical and mental health problems; however, several factors (e.g., age, gender, controllability of stressor) could moderate such associations (Zimmer-Gembeck \& Skinner, 2016). For example, Cabras and Mondo (2018) investigated Italian university freshmen and reported that males or younger students tended to avoid stressors and employ distractions. Additionally, the type of coping strategies individuals employ and the problems they are more likely to have can differ according to personality profiles (ConnorSmith \& Flachsbart, 2007; Kase et al., 2018; Steca et al., 2007). Given that personality and its related genetic factors could moderate the beneficial effects of psychological interventions (e.g., Bakermans-Kranenburg \& Van IJzendoorn, 2015; Pluess \& Boniwell, 2015), most of which include the session to improve one's coping skills (Amanvermez et al., 2020), considering individual differences in such factors would help psychologists develop more effective intervention programs, resulting in the promotion of participants' skills for coping with stressful events and/or negative emotions and preventing them from experiencing mental health problems.

Although an intervention in universities has an advantage, such as targeting many students at the same time (Cardemil \& Barber, 2001), it is difficult to develop and implement an individually tailored program because it is time intensive. Thus, it may be useful to classify participants into

\footnotetext{
${ }^{1}$ Problem-focused coping consists of strategies that attempt to modify and/or resolve stressful events, whereas emotion-focused coping refers to attempts to regulate one's emotional responses.

${ }^{2}$ Primary control coping consists of strategies that attempt to change the situation or emotions, whereas secondary control coping refers to attempts to adapt the stressor in order to fit the self to the environment (Connor-Smith \& Flachsbart, 2007).
} 
groups based on certain criteria and to plan and implement intervention programs considering the characteristics of each group (Kase et al., 2017).

\section{Sensory Processing Sensitivity as a Moderator of Psychological Interventions}

Recently, a growing number of studies have focused on an Environmental Sensitivity framework as a moderator of psychological interventions. Environmental Sensitivity describes how deeply individuals perceive and process physical, social, sensory, and internal stimuli (Greven et al., 2019; Pluess, 2015); that is, those with high sensitivity are more susceptible to both positive and negative environments in a for-better-and-for-worse manner than others (e.g., Belsky et al., 2007; Iimura, 2021; Lionetti et al., 2018; Pluess et al., 2020, August 19). A twin study involving 2,868 adolescents in the UK revealed that genetic factors could explain about $50 \%$ of the variance in Environmental Sensitivity and the only $22-35 \%$ variance of Big Five personality traits, indicating the high heritability of Environmental Sensitivity (Assary et al., 2020). Traditionally, sensitivity was assessed using genetic (e.g., dopamine receptor D2), physiological (e.g., cortisol reactivity), or psychological (e.g., negative emotionality) markers (Belsky \& Pluess, 2009). More recently, studies have demonstrated that Sensory Processing Sensitivity (SPS: Aron \& Aron, 1997), a temperament trait, is a potential marker of Environmental Sensitivity (e.g., Greven et al., 2019; Iimura \& Kibe, 2020; Pluess et al., 2020, August 19; Slagt et al., 2018). High-SPS individuals are characterized by stronger emotional reactivity, deeper processing of information, greater awareness of subtle stimuli, and being easily overstimulated (Aron et al., 2012; Homberg et al., 2016). To assess one's SPS level, two self-report measures are widely used: for adults, the Highly Sensitive Person Scale (HSPS; Aron \& Aron, 1997), and for children and adolescents, the Highly Sensitive Child Scale (Pluess et al., 2018).

In applied work, SPS has been shown to moderate the beneficial effects of psychological intervention programs. For instance, highly sensitive adolescents had lower depressive tendencies 
(Pluess \& Boniwell, 2015) or higher self-esteem (Kibe et al., 2020) than others after a school-based resilience program. Similarly, psychoeducational or physical exercise (i.e., yoga) programs implemented in educational institutions may have more beneficial effects for high-SPS students than low-SPS students, such as infrequent maladaptive behavior (Nocentini et al., 2018) or a low level of anxiety (Amemiya et al., 2020). Furthermore, in a questionnaire-based study, SPS moderated the relationships between life skills and depressive tendencies in university students; specifically, decision-making skills were effective for low-SPS individuals, but emotional coping skills were for high-SPS individuals (Yano et al., 2021a). These findings indicate that individual differences in SPS should be considered when constructing an intervention program to effectively promote coping skills and/or improve the mental health of university students.

Given that interventions conducted at universities often target many students at the same time (Kase et al., 2017), it could be useful to classify university students into groups based on SPS. Although the original study on SPS assumed that people fell into two groups (i.e., the $20 \%$ of highSPS individuals and the remaining 80\%) (Aron \& Aron, 1997), recent studies performed a series of latent class analyses and reported that people can be categorized into three sensitivity groups (i.e., about $30 \%$ of low-, about $40 \%$ of medium-, and about $30 \%$ of high-SPS individuals) (Lionetti et al., 2018; May et al., 2020; Pluess et al., 2018; Tillman et al., 2021; Yano \& Oishi, 2021). The characteristics of each group are often described using a flower metaphor: dandelions for low-SPS individuals because they can survive and thrive in whatever circumstances they encounter (i.e., they are not easily affected by an environment), orchids for those with high SPS because their survival and flourishing depend on conditions (i.e., they are easily affected by an environment), and tulips for individuals with medium SPS because they fall between dandelions and orchids in terms of their susceptibility to environments (Boyce \& Ellis, 2005; Lionetti et al., 2018; Pluess et al., 2018). Additionally, studies determined the preliminary cut-off scores to differentiate each group with 
good sensitivity and specificity (e.g., Lionetti et al., 2018; Pluess et al., 2018). Despite the risk that individuals might sometimes be miscategorized, the cut-off scores may provide useful information for smoothly classifying students in applied settings such as in clinics and education (Pluess et al., 2020, August 19; Yano et al., 2021a). Specifically, classifying participants into three sensitivity groups based on the cut-off scores helps psychologists construct intervention programs according to the characteristics of each group. Consequently, it can more effectively improve the mental health of university students. However, previous studies have not revealed the characteristics of each sensitivity group.

\section{Overview of the Present Study}

In summary, universities have recently conducted interventions to promote coping skills among university students, which are expected to improve their mental health. However, the approaches have several issues (i.e., much variation in effect sizes across studies, small-to-moderate effect sizes on average). To address these issues, considering the characteristics of the three sensitivity groups may improve the effectiveness of an intervention program. However, little is known about the characteristics of these sensitivity groups.

The present study aimed to explore the characteristics of the dispositional coping strategies (e.g., distraction, cognitive restructuring) employed by university students with low, medium, and high SPS and investigate the effectiveness thereof. Although studies suggest that strategies for improving mental health that are related to problem-focused coping are effective for low-SPS individuals and those related to emotion-focused coping are effective for high-SPS individuals (Brindle et al., 2015; Yano et al., 2021a), findings regarding the relationships between SPS and factors related to coping are limited in number. Therefore, it is difficult to form a clear hypothesis for this study. Additionally, because a quantitative method that uses scales to assess coping strategies (e.g., those used in former studies) may limit the findings we can obtain, this study 
employed an exploratory approach. Specifically, we conducted an open-ended question survey, which can be used to discover detailed characteristics beyond existing frameworks or theories (e.g., Khawaja \& Stallman, 2011), to extract the characteristics of the coping strategies used in the three sensitivity groups. Furthermore, two subgroups (i.e., better mental health, poorer mental health) were created within each sensitivity group, and we compared the characteristics between the subgroups. The results were expected to reveal effective coping strategies for better mental health among low-, medium-, and high-SPS university students. As such, they were expected to provide useful information for interventions at universities aimed at promoting coping skills and improving mental health.

\section{Methods}

\section{Participants and Procedures}

In this study, the Japanese research company Cross Marketing Inc. ${ }^{3}$ recruited participants and explained the purpose of the study to them. In total, 1,056 university students living in all of Japan's prefectures, all of whom voluntarily had been registered as Cross Marketing Inc.'s sample pool, provided their consent and participated in an anonymous online survey. However, 364 respondents were excluded because they did not follow the Directed Questions Scale (Maniaci \& Rogge, 2014) or provided an invalid answer to the open-ended question explained below. Thus, 692 participants were included in the statistical analyses (303 males and 389 females; $M_{\text {age }}=20.6$ years, $S D_{\text {age }}=1.4$ years). Of the participants, $12.4 \%$ were freshman, $33.8 \%$ second-year, $26.5 \%$ third-year, and $27.3 \%$ fourth-year students. As a reward, all participants were given points that could be exchanged for money with Cross Marketing Inc. The ethical board of XXX [blind] University approved this study

\footnotetext{
${ }^{3}$ Cross Marketing Inc. is one of the largest academic survey companies in Japan. At the time of the survey (June 2020), they had about 4.6 million web survey monitors all over Japan. Additionally, many researchers have collaborated with the company to collect data (e.g., Ueno et al., 2019; Yano et al., 2021a), suggesting the high quality and reliability of their data. Therefore, we decided to ask them to collect data for this study.
} 
(No. XXX).

\section{Measurements}

To assess SPS, we used the Japanese version of the 19-item HSPS (HSPS-J19; Takahashi, 2016). Although the original HSPS consisted of 27 items (Aron \& Aron, 1997), eight items were removed because of their low factor loadings in HSPS-J19 (Takahashi, 2016). As Lionetti et al. (2018) reported, the bi-factor structure in HSPS-J19 was best fitted to the data: three factors, i.e., ease of excitation (e.g., Do you get rattled when you have a lot to do in a short amount of time?), low sensory threshold (e.g., Are you bothered by intense stimuli, like loud noises or chaotic scenes?), and aesthetic sensitivity (e.g., Are you deeply moved by the arts or music?) in addition to the general sensitivity factor (e.g., Yano et al., 2021a). ${ }^{4}$ Furthermore, its validity has been confirmed by its correlations with personality constructs such as the Big Five traits (Takahashi, 2016; Yano et al., 2021b). Given the purpose of this study, we decided to use the total score of HSPS-J19. Each item was rated on a sevenpoint Likert-type scale ( 1 = "Strongly disagree" to 7 = "Strongly agree"), with higher scores indicating higher SPS. In this study, internal consistency was good $(a=.92)$.

To assess mental health, we used the Japanese version of the Kessler 10 (K10; Furukawa et al., 2008; Kessler et al., 2002). This scale consists of 10 items, with higher scores indicating poorer mental health (e.g., During the last 30 days, about how often did you feel tired out for no good reason?). Each item is rated on a five-point Likert-type scale $(0=$ "none of the time" to $4=$ "all of the time"). In this study, internal consistency was good $(a=.95) .^{5}$

To explore the coping strategies employed by our sample, an open-ended question was included in the questionnaire. Respondents were asked to answer the following question in Japanese:

\footnotetext{
${ }^{4}$ When we performed the confirmatory factor analysis, the bi-factor model showed better fit indices $(\mathrm{CFI}=.904$, $\mathrm{RMSEA}=.073, \mathrm{AIC}=44,462.695)$ than the three-factor model $(\mathrm{CFI}=.858, \mathrm{RMSEA}=.084, \mathrm{AIC}=44,796.207)$.

5 To confirm whether the use of the total score of K10 is statistically valid in this study, we performed a confirmatory factor analysis. The result demonstrated that our data was fitted to the one-factor model (CFI $=.979$, RMSEA $=.067)$.
} 
“When you face a stressful event, how do you usually deal with it?" Based on Uchida's (2018) study, we defined a stressful event as "a situation that is difficult or troubling for you, or that is frustrating or takes a lot of effort to deal with for you."

\section{Data Analysis}

First, the participants were classified into the three sensitivity groups, and two subgroups were created within each sensitivity group. Based on the cut-off scores of HSPS-J19 (Yano \& Oishi, 2021), those who scored from 19 to 69 were categorized into the low-SPS group, those who scored from 70 to 86 into the medium-SPS group, and those who scored from 87 to 133 into the high-SPS group. In addition, participants were further classified into the two subgroups based on the cut-off scores of the K10 (Furukawa et al., 2008). Those who scored 14 or less were categorized into the better mental health $(\mathrm{MH})$ subgroup, and those who scored more than 14 were classified into the poorer MH subgroup. The cut-off scores of both scales have good sensitivity and specificity; that is, they can correctly categorize individuals into groups according to level of SPS and mental health. Thus, classifying the participants into three sensitivity groups and creating two subgroups is acceptable (see Furukawa et al., 2008; Yano \& Oishi, 2021). Second, a chi-square test was performed to investigate whether any bias existed in the ratio of each group and subgroup.

Third, to analyze the text data statistically, we performed a quantitative text analysis. This analysis automatically extracts components (i.e., words, phrases) from the data and statistically explores the whole picture of the data, which could reveal participants' characteristics with little prejudice on the side of the researcher (Higuchi, 2016, 2017). In the pre-processing phase, we carefully checked the raw data followed by correcting any spelling errors and omissions, replacing words with the same meaning (e.g., "tomodachi" was replaced with "yu-jin" in Japanese because both words mean "friend" in English), and sorting compound words. After confirming frequently appearing components, a co-occurrence network analysis was performed. This analysis depicts the 
set of words (and some grouping variables) that frequently appear together and links a component to another component or a grouping variable based on Jaccard indices (e.g., Higuchi, 2017; Vallejomedina et al., 2020). Furthermore, visualizing the co-occurrence network advances our understanding of the results (Corman et al., 2002). Because of its advantages, recent studies in the field of psychology have employed co-occurrence network analyses (e.g., Kase et al., 2016; Kase et al., 2020; Moeller et al., 2018; Vallejo-Medina et al., 2020). Given its purpose, this study performed the analysis, using the two subgroups (i.e., better $\mathrm{MH}$ and poorer $\mathrm{MH}$ ) as a grouping variable, by each sensitivity group (i.e., low-, medium-, high-SPS group). This procedure enabled us to compare the characteristics of the coping strategies used by the university students with better and poorer MH in each sensitivity group.

To ensure validity, the four researchers discussed the results. Specifically, the first author of this article interpreted the results of the co-occurrence network analysis by reviewing the raw data. Next, the three co-authors from a variety of research backgrounds (i.e., sport psychology, social psychology, health psychology) ${ }^{6}$ checked whether the interpretation depended on the first author's prejudice. When one or more co-author(s) believed the first author's prejudice was evident, it was improved through discussion among the four researchers until consensus was reached. In the cooccurrence network analysis, depicting too many or too few components make it difficult to interpret the results (Higuchi, 2017; Vallejo-Medina et al., 2020). Based on previous studies performing a similar analysis (Kase et al., 2016; Moeller et al., 2018), we included a maximum of 50 components in the analysis. This study performed a chi-square test using the free software HAD 17.0 (Shimizu, 2016) and the quantitative text analysis using KH Coder 3 (Higuchi, 2016, 2017).

\footnotetext{
${ }^{6}$ To enhance general objectivity as much as possible, the results were discussed with multiple researchers from a variety of research backgrounds (e.g., Carter et al., 2014).
} 


\section{Results}

\section{Classification of Participants}

In this study, participants were classified based on the cut-off scores of the HSPS-J19 (Yano \& Oishi, 2021) and K10 (Furukawa et al., 2008). In total, 241 participants (34.8\%) belonged to the low-SPS group (better MH: $n=209$; poorer MH: $n=32), 266(38.4 \%)$ to the medium-SPS group (better MH: $n=183$; poorer MH: $n=83)$, and the remaining $185(26.8 \%)$ to the high-SPS group (better MH: $n=54$; poorer MH: $n=131$ ). Because the chi-square value was significant $\left(\chi^{2}=154.8\right.$, $d f=2, p<.001$; Cramer's $V=.47,95 \%$ CI $[.40, .55])$, a residual analysis was performed. The results indicated that more participants belonged to the low-SPS group with better MH or to the high-SPS group with poorer MH. On the other hand, fewer participants belonged to the low-SPS group with poorer MH or the high-SPS group with better $\mathrm{MH}$.

\section{Quantitative Text Analyses for the Open-Ended Question}

Components in the entire sample that appeared frequently were identified (Table 1), followed by the co-occurrence network analysis with the two grouping variables (i.e., better $\mathrm{MH}$, poorer $\mathrm{MH}$ ) by each sensitivity group.

[Table 1]

Low-SPS Group. Figure 1 displays the co-occurrence network for the low-SPS group. As mentioned in the Data Analysis section, we decided to include 50 components at maximum. Therefore, in this analysis, components that appeared in the data more than six times were used. Both subgroups were linked to components such as "resolve" (e.g., I resolve it as quickly as possible), "escape" (e.g., I escape from reality), "positive" (e.g., I think as positively as possible), "like" (e.g., I involve myself fully in the things I like), and "share my concerns" (e.g., I share my concerns with trustworthy people). Therefore, low-SPS university students, regardless of their mental health level, were interpreted as coping with stress by employing strategies such as Problem solving, Avoidance, Positive thinking, 
Distraction, and Sharing one's concerns with others. The better MH subgroup was linked to components such as "calm down" (e.g., I take a moment to calm down), "music" (e.g., I listen to music), "reflect" (e.g., I reflect that this is an opportunity), and "friends" (e.g., I talk with friends). Therefore, low-SPS university students with better MH were interpreted as coping with stress by employing strategies such as Emotional regulation, Distraction, and Emotional and instrumental support seeking to friends. Finally, the poorer MH subgroup was linked to components such as "hobby" (e.g., I immerse myself in a hobby as a diversion), "occurrence" (e.g., I don't directly face the occurrences), and "alone” (e.g., I cry alone so that I don't bother the people around me). Therefore, low-SPS university students with poorer MH were interpreted as coping with stress by employing strategies such as Distraction, Avoidance, and Withdrawal.

[Figure 1]

Medium-SPS Group. Figure 2 displays the co-occurrence network for the medium-SPS group. As mentioned above, we decided to include 50 components at maximum, so in the analysis, components that appeared in the data more than seven times were used. Both subgroups were linked to components such as "resolve" (e.g., I resolve it as quickly as possible), "escape" (e.g., I escape from reality), "like" (e.g., I do things I like), "share my concerns" (e.g., I share my concerns with trustworthy people), and "calm down" (e.g., I calm down by taking deep breaths). Therefore, mediumSPS university students, regardless of their mental health level, were interpreted as coping with stress by employing strategies such as Problem solving, Avoidance, Distraction, Sharing one's concerns with others, and Emotional regulation. The better MH subgroup was linked to components such as "divert" (e.g., I do something else to divert my attention), "avoid" (e.g., If I can avoid it, I do), "positive" (e.g., I think positively about good experiences), and "friends" (e.g., I ask for help from friends). Therefore, medium-SPS university students with better MH were interpreted as coping with stress by employing strategies such as Distraction, Avoidance, Positive thinking, and Instrumental 
support seeking to friends. Finally, the poorer MH subgroup was linked to components such as "refresh" (e.g., I refresh myself by watching videos and so on), "talk" (e.g., I try talking with my family), "cry" (e.g., I cry my heart out when no one else is around), and "effort" (e.g., I think about the cause and make an effort to resolve it). Therefore, medium-SPS university students with poorer MH were interpreted as coping with stress by employing strategies such as Distraction, Emotional support seeking to others, Withdrawal, and Cause analysis and information gathering.

[Figure 2]

High-SPS Group. Figure 3 displays the co-occurrence network for the high-SPS group. In order to not exceed the 50 components included in this analysis, components that appeared in the data more than six times were used. Both subgroups were linked to components such as "resolve" (e.g., I take action to resolve things, even if only partially), “overthink” (e.g., I make sure I don't overthink), "like" (e.g., I do things I like), and "share my concerns" (e.g., I share my concerns with others). Therefore, high-SPS university students, regardless of their mental health level, were interpreted as coping with stress by employing strategies such as Problem solving, Avoidance, Distraction, and Sharing one's concerns with others. The better MH subgroup was linked to components such as "positive" (e.g., I think as positively as possible), "cry" (e.g., I cry my heart out or take it out on objects), “calm down" (e.g., I let time pass and wait until my feelings calm down), and "talk" (e.g., I talk with people who understand me). Therefore, high-SPS university students with better MH were interpreted as coping with stress by employing strategies such as Positive thinking, Emotional expression, Emotional regulation, and Emotional support seeking to others. Finally, the poorer $\mathrm{MH}$ subgroup was linked to components such as "hobby" (e.g., I immerse myself in a hobby as a diversion), "escape" (e.g., I take a moment to escape from the situation), "cause" (e.g., I cope with stress by thinking about the cause), and "endure" (e.g., I endure it until things are better). Therefore, high-SPS university students with poorer MH were interpreted as coping with stress by employing 
strategies such as Distraction, Temporary escape, Cause analysis and information gathering, and Endurance.

\section{[Figure 3]}

The Jaccard indices, the degree to which a component and a grouping variable co-occur, are reported in the Supporting Information (see Table S2).

\section{Discussion}

This study classified Japanese university students into three sensitivity groups and compared the characteristics of the coping strategies of the two subgroups in each sensitivity group. The results are expected to provide useful evidence for university mental health professionals to create intervention programs considering individual differences in SPS to effectively promote coping skills and consequently improve mental health. The co-occurrence network analyses in this study suggested that while some coping strategies (i.e., Problem solving, Avoidance, Distraction, and Sharing one's concerns with others) were common to all the sensitivity groups and subgroups, others were specific to a certain sensitivity group and/or subgroup (Table 2). Therefore, effective coping strategies for better mental health could differ according to the level of SPS.

[Table 2]

\section{Relationship between SPS and Mental Health}

The chi-square test and residual analysis showed that university students with high SPS tend to have poorer MH. Conversely, low-SPS students are more likely to have better MH. These relationships between SPS and mental health support previous findings that SPS, at least when assessed using HSPS(-J19), is positively correlated with negative affect (e.g., Lionetti et al., 2019; Yano et al., 2021a). 


\section{Effective Coping Strategies for Low-, Medium-, and High-SPS University Students}

First, for low-SPS university students, coping strategies such as Emotional regulation and Emotional and instrumental support seeking to friends were related to better mental health. The strategies identified in this study correspond to the primary control framework (Compas et al., 2017; Connor-Smith et al., 2000; Connor-Smith \& Flachsbart, 2007), which refers to "active attempts to control or change a bad situation or one's emotional reaction to the situation" (Connor-Smith \& Flachsbart, 2007, p.1082). University students with low SPS have been reported to possess higher skills in coping with negative emotions (Brindle et al., 2015; Yano et al., 2021a). Therefore, discovering strategies such as Emotional regulation and Emotional support seeking to friends provides valuable insight into the existing evidence. Furthermore, a recent study showed that higher decision-making skills (e.g., planning, knowledge summarization) were correlated with reduced depressive tendencies in low-SPS university students (Yano et al., 2021a). Given that Instrumental support seeking to friends was discovered in this study, they may make the best decisions based on multiple perspectives by asking their friends for help and/or advice. Yano et al. (2021a) inferred that low-SPS individuals make decisions without sufficient caution (Aron et al., 2012), resulting in increased depressive tendencies and/or maladaptive behavior. Additionally, when experiencing negative emotions like anxiety, people often engage in task-irrelevant information (e.g., Eysenck, 1979), which may result in making a mistake and undesirable consequences. Taken together, advice and/or help from friends after coping with negative emotions might promote appropriate decision making, resulting in better MH for low-SPS university students. However, the temporal relationships between the strategies should be examined.

Second, for medium-SPS university students, coping strategies such as Positive thinking and Instrumental support seeking to friends were related to better mental health. The Instrumental support seeking to friends strategy corresponds with primary control, and that of Positive thinking corresponds 
with secondary control (Compas et al., 2017; Connor-Smith \& Flachsbart, 2007; Zimmer-Gembeck \& Skinner, 2016), which refers to "attempts to adapt to a stressor to create a better fit between the self and the environment" (Connor-Smith \& Flachsbart, 2007, p.1082). The effective strategies for medium-SPS university students differed slightly from those used by low-SPS students, although both groups employed Instrumental support seeking to friends. Given that the strategies classified in the secondary control framework are employed when primary control strategies cannot change the situation or one's emotions (Rothbaum et al., 1982), the following temporal sequence might be hypothesized: medium-SPS university students may attempt to solve a problem with support from friends, but if this fails, Positive thinking, a kind of cognitive reappraisal, may be employed.

Finally, for high-SPS university students, coping strategies such as Positive thinking, Emotional expression, Emotional regulation, and Emotional support seeking to friends were related to better mental health. As mentioned above, the strategies of Emotional regulation and Emotional support seeking to friends correspond to primary control, that of Positive thinking corresponds to secondary control (e.g., Compas et al., 2017; Connor-Smith \& Flachsbart, 2007; Zimmer-Gembeck \& Skinner, 2016). Additionally, studies have considered how Emotional expression corresponds to a primary control framework (Compas et al., 2017; Zimmer-Gembeck \& Skinner, 2016) or negative emotion-focused coping, which refers to "emotional regulation and expression strategies that suggest loss of control (e.g., hitting, throwing objects), distress (e.g., crying, yelling, self-blame), or hostility toward others" (Connor-Smith \& Flachsbart, 2007, p.1082). All the strategies identified in the highSPS university students are related to the control of negative emotion, rather than an approach to stressors. Note that problem-oriented (i.e., Instrumental support seeking to friends) and emotionoriented strategies were found for low- and medium-SPS university students. Because highly sensitive individuals tend to feel more negative emotions when exposed to aversive stimuli (Aron et al., 2012; Homberg et al., 2016; Pluess, 2015), higher skills in coping with emotion could play an 
important role in decreasing their depressive tendencies (Brindle et al., 2015; Yano et al., 2021a). Our results may provide insight into how high-SPS university students can cope with negative emotions. According to a primary/secondary control framework (Rothbaum et al., 1982), high-SPS university students firstly may attempt to control their negative emotions by taking a moment to calm down, seeking emotional support, and/or emotional expression. However, if the strategies fail, they think about the situation positively.

Interestingly, some of our findings are inconsistent with existing evidence. A recent review reported that emotional support was associated with negative consequences (Zimmer-Gembeck \& Skinner, 2016), which was supported only for medium-SPS university students in this study. For lowand high-SPS university students, seeking emotional support might contribute to better $\mathrm{MH}$. However, the moderation effect of SPS on the relationship between emotional support and MH should be further investigated. Conversely, a Cause analysis and information gathering strategy may be related to poorer mental health for medium- and high-SPS university students. A recent study pointed out that employing this strategy results in personal growth in some cases, but promotes more negative rumination in others (Kamijo \& Yukawa, 2016). Given the findings of this study, SPS might also moderate the relationship between this strategy and mental health; however, further investigation is needed.

\section{Strengths, Limitations, and Future Directions}

This study performed a series of quantitative text analyses and provided findings regarding effective coping strategies for better mental health in low-, medium-, and high-SPS university students. The results support the existing evidence that individual differences in SPS should be considered even when constructing a universally designed intervention program (Kibe et al., 2020; Pluess \& Boniwell, 2015; Yano et al., 2021a). In practice or in applied research, programs considering the characteristics of each sensitivity group could more effectively improve mental health than those 
that are universally designed, which are traditionally conducted. For example, it may be important for high-SPS university students to increase the variation in how to control their negative emotions, whereas perceiving support from friends and/or changing one's cognition to become more positive may be important for low- and medium-SPS students.

Despite its advantages, there are several limitations to this study. First, we focused on dispositional coping strategies. It is well known that the moderators of the relationships between a specific coping strategy and health outcomes include not only several personality factors (e.g., SPS, Big Five traits), but also the type of stressor (e.g., duration, magnitude) (Penley et al., 2002). Because there is little evidence of the characteristics of each sensitivity group, we explored the dispositional coping strategies employed in participants' daily lives (i.e., they were not asked to answer regarding a specific situation). Thus, this study did not control for the type of stressor. To elaborate our findings, the changes in several factors across time should be captured controlling for the type of stressor (e.g., interpersonal stress, job hunting).

Second, more factors should be considered in order to enhance the generalizability of our results. For example, the type of stressors one is more likely to encounter and/or the ways of coping with them could differ according to age (Cabras \& Mondo, 2018) and academic year (Iorga et al., 2018). Another example is cultural factors; in particular, a recent study suggested that the association of coping strategies with depression or somatic symptoms slightly differed between Japanese and American university students (Hamamura \& Mearns, 2019). Therefore, future studies that consider these factors, such as a cross-cultural design, should provide further insights.

Third, this study analyzed text data obtained from a cross-sectional survey. Analyses of qualitative data, including text, risk involving the subjectivity of the researchers, which is not a risk with quantitative data (even though consensus among four researchers with different areas expertise does somewhat enhance the objectivity of the results). Additionally, we used SPS and MH as grouping 
variables in the co-occurrence network analysis, though they are continuous in nature. For this reason, it should be noted that the generalizability of our findings is somewhat limited. Further investigation applying a quantitative approach is also needed. Moreover, revealing the temporal sequence in which each strategy is employed, as was inferred above, provides useful evidence for practice.

Fourth and finally, this study could not reveal why there were variations in the number of effective coping strategies in the three sensitivity groups. Whereas only the strategies corresponding to the primary control framework were extracted in the low-SPS group, those corresponding to primary and secondary control (plus negative emotion-focused) coping were extracted in the highSPS group. An important issue is to investigate whether such variations are derived from withinindividual (i.e., richness of the coping strategies an individual can employ) or between-individual factors. If it is derived from within-individual factors, future studies should consider the concepts that were not focused on in this study, such as coping flexibility (e.g., Kato, 2012). Addressing the abovementioned limitations could provide more information for interventions to ensure they effectively improve the mental health of university students. 


\section{References}

Acharya, L., Jin, L., \& Collins, W. (2018). College life is stressful today-emerging stressors and depressive symptoms in college students. Journal of American College Health, 66, 655664.

Amanvermez, Y., Rahmadiana, M., Karayotaki, E., De Wit, L., Ebert, D. D., Kessler, R. C., Cuijpers, P. (2020). Stress management interventions for college students: A systematic review and meta-analysis. Clinical Psychology: Science and Practice, e12342.

Amemiya, R., Takahashi, G., Rakwal, R., Kahata, M., Isono, K., \& Sakairi, Y. (2020). Effects of yoga in a physical education course on attention control and mental health among graduate students with high sensory processing sensitivity. Cogent Psychology, 7, 1778895.

American College Health Association. (2009). American College Health Association-National college health assessment Spring 2008 reference group data report (abridged): The American College Health Association. Journal of American College Health, 57, 477-488.

Andrews, B., \& Wilding, J. M. (2004). The relation of depression and anxiety to life-stress and achievement in students. British Journal of Psychology, 95, 509-521.

Aron, E. N., \& Aron, A. (1997). Sensory-processing sensitivity and its relation to introversion and emotionality. Journal of Personality and Social Psychology, 73, 345-368.

Aron, E. N., Aron, A., \& Jagiellowicz, J. (2012). Sensory processing sensitivity: A review in the light of the evolution of biological responsivity. Personality and Social Psychology Review, $16,262-282$.

Assary, E., Zavos, H. M. S., Krapohl, E., Keers, R., \& Pluess, M. (2020). Genetic architecture of Environmental Sensitivity reflects multiple heritable components: A twin study with adolescents. Molecular Psychiatry. https://doi.org/10.1038/s41380-020-0783-8 
Auerbach, R. P., Alonso, J., Axinn, W. G., Cuijipers, P., Ebert, D. D., Green, J. G., Hwang, I., Kessler, R. C., Liu, H., Mortier, P., Nock, M. K., Pinder-Amaker, S., Sampson, N. A., Aguilar-Gaxiola, S., Al-Hamzawi, A., Andrade, L. H., Benjet, C., Caldas-de-Almeida, J. M., Demyttenaere, K., ... Bruffaerts, R. (2016). Mental disorders among college students in the WHO World Mental Health Surveys. Psychological Medicine, 46, 2955-2970.

Bakermans-Kranenburg, M. J., \& Van IJzendoorn, M. H. (2015). The hidden efficacy of intervention: Gene $\times$ environment experiments from a differential susceptibility perspective. Annual Review of Psychology, 66, 381-409.

Belsky, J., Bakermans-Kranenburg, M. J., \& Van IJzendoorn, M. H. (2007). For better and for worse: Differential susceptibility to environmental influences. Current Directions in Psychological Science, 16, 300-304.

Belsky, J., \& Pluess, M. (2009). Beyond diathesis stress: Differential susceptibility to environmental influences. Psychological Bulletin, 135, 885-908.

Boyce, T. W., \& Ellis, B. J. (2005). Biological sensitivity to context: I. An evolutionarydevelopmental theory of the origins and functions of stress reactivity. Development and Psychopathology, 17, 271-301.

Brindle, K., Moulding, R., Bakker, K., \& Nedeljkovic, M. (2015). Is the relationship between sensory-processing sensitivity and negative affect mediated by emotional regulation? Australian Journal of Psychology, 67, 214-221.

Cabras, C., \& Mondo, M. (2018). Coping strategies, optimism, and life satisfaction among first-year university students in Italy: Gender and age differences. Higher Education, 75, 643-654.

Cardemil, E. V., \& Barber, J. P. (2001). Building a model for prevention practice: Depression as an example. Professional Psychology: Research and Practice, 32, 392-401. 
Carter, N., Bryant-Lukosius, D., DiCenso, A., Blythe, J., \& Neville, A. J. (2014). The use of triangulation in qualitative research. Oncology Nursing Forum, 41, 545-547.

Compas, B., Jaser, S., Bettis, A., Watson, K., Gruhn, M., Dunbar, J., Williams, E., \& Thigpen, J. (2017). Coping, emotion regulation and psychopathology in childhood and adolescence: A meta-analysis and narrative review. Psychological Bulletin, 143, 939-991.

Connor-Smith, J., Compas, B., Wadsworth, M., Thomsen, A., \& Saltzman, H. (2000). Response to stress in adolescence: Measurement of coping and involuntary stress responses. Journal of Consulting and Clinical Psychology, 68, 976-992.

Connor-Smith, J., \& Flachsbart, C. (2007). Relations between personality and coping: A metaanalysis. Journal of Personality and Social Psychology, 93, 1080-1107.

Corman, S. R., Kuhn, T., McPhee, R. D., \& Dooley, K. (2002). Studying complex discursive systems: Centering reasonance analysis of communication. Human Communication Research, 28, 157-206.

Eysenck, M. W. (1979). Anxiety, learning, and memory: A reconceptualization. Journal of Research in Personality, 13, 363-385.

Furukawa, T. A., Kawakami, N., Saitoh, M., Ono, Y., Nakane, Y., Nakamura, Y., Tachimori, H., Iwata, N., Uda, H., Nakane, H., Watanabe, M., Naganuma, Y., Hata, Y., Kobayashi, M., Miyake, Y., Takeshima, T., \& Kikkawa, T. (2008). The performance of the Japanese version of the K6 and K10 in the World Mental Health Survey Japan. International Journal of Methods in Psychiatric Research, 17, 152-158.

Greven, C., Lionetti, F., Booth, C., Aron, E. N., Fox, E., Schendan, H. E., Pluess, M., Bruining, H., Acevedo, B., Bijttebier, P., \& Homberg, J. (2019). Sensory processing sensitivity in the context of environmental sensitivity: A critical review and development of research agenda. Neuroscience and Biobehavioral Reviews, 98, 287-305. 
Hamamura, T., \& Mearns, J. (2019). Depression and somatic symptoms in Japanese and American college students: Negative mood regulation expectancies as a personality correlate. International Journal of Psychology, 54, 351-359.

Higuchi, K. (2016). A two-step approach to quantitative content analysis: KH Coder tutorial using Anne of Green Gables (Part I). Ritsumeikan Social Sceinces Review, 52(3), 77-91.

Higuchi, K. (2017). A two-step approach to quantitative content analysis: KH Coder tutorial using Anne of Green Gables (Part II). Ritsumeikan Social Sceinces Review, 53(1), 137-147.

Homberg, J., Schubert, D., Asan, E., \& Aron, E. N. (2016). Sensory processing sensitivity and serotonin gene variance: Insights into mechanisms shaping environmental sensitivity. Neuroscience and Biobehavioral Reviews, 71, 472-483.

Iimura, S. (2021). Highly sensitive adolescents: The relationship between weekly life events and weekly socioemotional well-being. British Journal of Psychology. Advance Online Publication. https://doi.org/10.1111/bjop.12505

Iimura, S., \& Kibe, C. (2020). Highly sensitive adolescent benefits in positive school transitions: Evidence for vantage sensitivity in Japanese high-schoolers. Developmental Psychology, 56, $1565-1581$.

Iorga, M., Dondas, C., Zugun-Eloae, C. (2018). Depressed as freshman, stressed as seniors: The relationship between depression, perceived stress and academic results among medical students. Behavioral Sciences, 8, 70. https://doi.org/10.3390/bs8080070

Kamijo, N., \& Yukawa, S. (2016). The role of intrusive and deliberate ruminations for meaning making in stressful events. Japanese Journal of Psychology, 86, 513-523.

Kase, T., Bannai, K., \& Oishi, K. (2016). Behavior and thought constructing life skills in Japanese adults: An exploratory study using quantitative text analysis. Japanese Journal of Social Psychology, 32, 60-67. 
Kase, T., Ueno, Y., Kajiuchi, D., \& Shimamoto, K. (2018). Characteristics of Resilients, Overcontrollers, Undercontrollers, and the other type in personality prototypes: Based on comparison of the levels of life skills. Japanese Journal of Personality, 27, 164-167.

Kase, T., Ueno, Y., \& Oishi, K. (2017). Relationship between the classification of Japanese college students based on personality prototypes and mental health. Japanese Journal of Education and Health Promotion, 25, 195-203.

Kase, T., Ueno, Y., Shimamoto, K., \& Oishi, K. (2020). Characteristics of behavior and thinking related to problem-solving in the daily lives of persons with a high sense of coherence: A qualitative study using quantitative text analysis. Stress Science Research. Advance Online Publication. https://doi.org/10.5058/stresskagakukenkyu.2020001

Kato, T. (2012). Development of the Coping Flexibility Scale: Evidence for the coping flexibility hypothesis. Journal of Counseling Psychology, 59, 262-273.

Kessler, R. C., Andrews, G., Colpe, L. J., Hiripi, E., Mroczek, D. K., Normand, S. L. T., Walters, E. E., \& Zaslavsky, A. M. (2002). Short screening scales to monitor population prevalences and trends in non-specific psychological distress. Psychological Medicine, 32, 959-976.

Khawaja, N., \& Stallman, H. (2011). Understanding the coping strategies of international students: A qualitative approach. Journal of Psychologists and Counsellors in Schools, 21, 203-224.

Kibe, C., Suzuki, M., Hirano, M., \& Boniwell, I. (2020). Sensory processing sensitivity and culturally modified resilience education: Differential susceptibility in Japanese adolescents. PLoS ONE, 15(9), e0239002.

Lazarus, R. S., \& Folkman, S. (1984). Stress, appraisal, and coping. Springer.

Lionetti, F., Aron, A., Aron, E. N., Burns, G. L., Jagiellowicz, J., \& Pluess, M. (2018). Dandelions, tulips and orchids: Evidence for the existence of low-sensitive, medium-sensitive, and highsensitive individuals. Translational Psychiatry, 8, 24. 
Lionetti, F., Pastore, M., Moscardino, U., Nocentini, A., Pluess, K., \& Pluess, M. (2019). Sensory processing sensitivity and its association with personality traits and affect: A meta-analysis. Journal of Research in Personality, 81, 138-152.

Maniaci, M. R., \& Rogge, R. D. (2014). Caring about carelessness: Participant inattention and its effects on research. Journal of Research in Personality, 48, 61-83.

May, A. K., Norris, S. A., Richter, L. M., \& Pitman, M. M. (2020). A psychometric evaluation of the Highly Sensitive Person Scale in ethnically and culturally heterogeneous South African samples. Current Psychology. Advance Online Publication. https://doi.org/10.1007/s12144020-00988-7

Moeller, J., Ivcevic, Z., Brackett, M. A., \& White, A. E. (2018). Mixed emotions: Network analyses of intra-individual co-occurrences within and across situations. Emotion, 18, 1106-1121.

Mortier, P., Cuijpers, P., Kiekens, G., Auerbach, P., Demyttenaere, K., Green, J. G., Kessler, R. C., Nock, M. K., \& Bruffaerts, R. (2018). The prevalence of suicidal thoughts and behaviours among college students: A meta-analysis. Psychological Medicine, 48, 554-565.

Nippon Foundation Suicide Prevention Project. (2019). The Nippon Foundation Suicide Awareness Survey 2016. Retrieved from https://www. nippon-foundation.or.jp/app/uploads/2019/03/ wha_pro_sui_mea_11-1.pdf (October 8, 2020)

Nocentini, A., Menesini, E., \& Pluess, M. (2018). The personality trait of environmental sensitivity predicts children's positive response to school-based antibullying intervention. Clinical Psychological Science, 6, 848-859.

Pascoe, M. C., Hetrick, S. E., \& Parker, A. G. (2020). The impact of stress on students in secondary school and higher education. International Journal of Adolescence and Youth, 25, 104-112. 
Penley, J. A., Tomaka, J., \& Wiebe, J. S. (2002). The association of coping to physical and psychological health outcomes: A meta-analytic review. Journal of Behavioral Medicine, $25,551-603$.

Pluess, M. (2015). Individual differences in environmental sensitivity. Child Development Perspectives, 9, 138-143.

Pluess, M., Assary, E., Lionetti, F., Lester, K. J., Krapohl, E., Aron, E. N., \& Aron, A. (2018). Environmental sensitivity in children: Development of the Highly Sensitive Child Scale and identification of sensitivity groups. Developmental Psychology, 54, 51-70.

Pluess, M., \& Boniwell, I. (2015). Sensory-processing sensitivity predicts treatment response to a school-based depression prevention program: Evidence of vantage sensitivity. Personality and Individual Differences, 82, 40-45.

Pluess, M., Lionetti, F., Aron, E. N., \& Aron, A. (2020, August 19). People differ in their sensitivity to the environment: An integrated theory and empirical evidence. PsyArxiv. https://doi.org/10.31234/osf.io/w53yc

Rothbaum, F., Weisz, J. R., \& Snyder, S. S. (1982). Changing the world and changing the self: A two-process model of perceived control. Journal of Personality and Social Psychology, 42, $5-37$.

Shimizu, H. (2016). An introduction to the statistical free software HAD: Suggestions to improve teaching, learning and practice data analysis. Journal of Media, Information and Communication, 1, 59-73.

Slagt, M., Dubas, J. S., van Aken, M. A., Ellis, B. J., Deković, M. (2018). Sensory processing sensitivity as a marker of differential susceptibility to parenting. Developmental Psychology, $54,543-558$. 
Steca, P., Alessandri, G., Vecchio, G. M., \& Caprara, G. V. (2007). Being a successful adolescent at school and with peers. The discriminative power of a typological approach. Emotional and Behavioral Difficulties, 12, 147-162.

Takahashi, A. (2016). Development of Japanese version of the 19-item Highly Sensitive Person Scale (HSPS-J19). Japanese Journal of Research on Emotions, 23, 68-77.

Tillman, T., Bertrams, A., El Matany, K., \& Lionetti, F. (2021). Replication of the existence of three sensitivity groups in a sample of German adolescents. European Journal of Developmental Psychology, 18, 131-143.

Ueno, Y., Takahashi, A., \& Ueno, Y. (2019). Relationship between sensory-processing sensitivity and age in a large cross-sectional Japanese sample. Heliyon, 5, e02508.

Uchida, K. (2018). Effects of dispositional emotion-focused coping on daily mood. Japanese Journal of Personality, 27, 1-11.

Vallejo-Medina, P., Correa, J. C., Gómez-Lugo, M., Saavedra-Roa, D. A., García-Montaño, E., Pérez-Pedraza, D., Niebles-Charris, J., García-Roncallo, P., Abello-Luque, D., Espada, J. P., \& Morales, A. (2020). A text mining approach for adapting a school-based sexual health promotion program in Colombia. Preventive Medicine Reports, 18, 101090.

Werner-Seidler, A., Perry, Y., Calear, A. L., Newby, J. M., \& Christensen, H. (2017). School-based depression and anxiety prevention programs for young people: A systematic review and meta-analysis. Clinical Psychology Review, 51, 30-47.

Yano, K., Kase, T., \& Oishi, K. (2021a). Sensory processing sensitivity moderates the relationships between life skills and depressive tendencies in university students. Japanese Psychological Research, 63, 152-163. 
Yano, K., Kase, T., \& Oishi, K. (2021b). The associations between sensory processing sensitivity and the Big Five personality traits in a Japanese sample. Journal of Individual Differences, $42,84-90$.

Yano, K., \& Oishi, K. (2021). Replication of the three sensitivity groups and investigation of their characteristics in Japanese samples. Current Psychology. Advance Online Publication. https://doi.org/10.1007/s12144-021-01537-6

Zimmer-Gembeck, M. J., \& Skinner, E. A. (2016). The development of coping: Implications for psychopathology and resilience. In Cicchetti, D. (Ed.), Developmental psychology: Risk, resilience, and intervention (pp. 485-545). Wiley. https://doi.org/10.1002/9781119125556.devpsy410 
Table 1. Frequently appearing components in the entire sample

\begin{tabular}{|c|c|c|c|}
\hline Component & Frequency & Component & Frequency \\
\hline Think & 251 & Watch & 39 \\
\hline Myself & 121 & Eat/Something else & 36 \\
\hline Possible & 120 & A moment & 32 \\
\hline Stress & 105 & Positive & 30 \\
\hline Resolve & 90 & Reflect & 29 \\
\hline Sleep & 87 & Somehow/Good/Talk & 28 \\
\hline Overthink & 61 & Action & 27 \\
\hline Music & 60 & Way & 26 \\
\hline Like & 59 & Time & 25 \\
\hline People & 53 & Alone/Divert & 24 \\
\hline Cause/Listen & 50 & Release & 23 \\
\hline Cope/Forget & 49 & Cry & 21 \\
\hline Share my concerns & 46 & Hobby/Get over & 20 \\
\hline Calm down & 44 & Deep/Effort/Feelings & 19 \\
\hline Occurrence/Escape & 42 & Endure/Avoid/Fun & 18 \\
\hline Attention/Friends & 40 & Refresh/Bit by bit/Conditions/Look for & 16 \\
\hline Votes. In our sample, th & 1 number 0 & of automatically extracted components & was 11,336 \\
\hline
\end{tabular}


Table 2. Characteristics of coping strategies in the three sensitivity groups

\begin{tabular}{|c|c|c|c|}
\hline & $\begin{array}{l}\text { Specific to the better } \mathrm{MH} \\
\text { subgroup }\end{array}$ & $\begin{array}{l}\text { Specific to the poorer } \mathrm{MH} \\
\text { subgroup }\end{array}$ & Common to both subgroups \\
\hline Low-SPS & $\begin{array}{l}\text { Emotional regulation, } \\
\text { Emotional and } \\
\text { instrumental support } \\
\text { seeking to friends }\end{array}$ & Withdrawal & $\begin{array}{l}\text { Problem solving, Avoidance, } \\
\text { Positive thinking, Distraction, } \\
\text { Sharing one's concerns to } \\
\text { others }\end{array}$ \\
\hline Medium-SPS & $\begin{array}{l}\text { Positive thinking, } \\
\text { Instrumental support } \\
\text { seeking to friends }\end{array}$ & $\begin{array}{l}\text { Emotional support } \\
\text { seeking to others, } \\
\text { Withdrawal, } \\
\text { Cause analysis and } \\
\text { information gathering }\end{array}$ & $\begin{array}{l}\text { Problem solving, Avoidance, } \\
\text { Distraction, Sharing one's } \\
\text { concerns to others, } \\
\text { Emotional regulation }\end{array}$ \\
\hline High-SPS & $\begin{array}{l}\text { Positive thinking, } \\
\text { Emotional expression, } \\
\text { Emotional regulation, } \\
\text { Emotional support } \\
\text { seeking to others }\end{array}$ & $\begin{array}{l}\text { Temporal avoidance, } \\
\text { Endurance, } \\
\text { Cause analysis and } \\
\text { information gathering }\end{array}$ & $\begin{array}{l}\text { Problem solving, Avoidance, } \\
\text { Distraction, Sharing one's } \\
\text { concerns to others }\end{array}$ \\
\hline
\end{tabular}

Notes. $\mathrm{MH}=$ mental health, $\mathrm{SPS}=$ Sensory Processing Sensitivity 


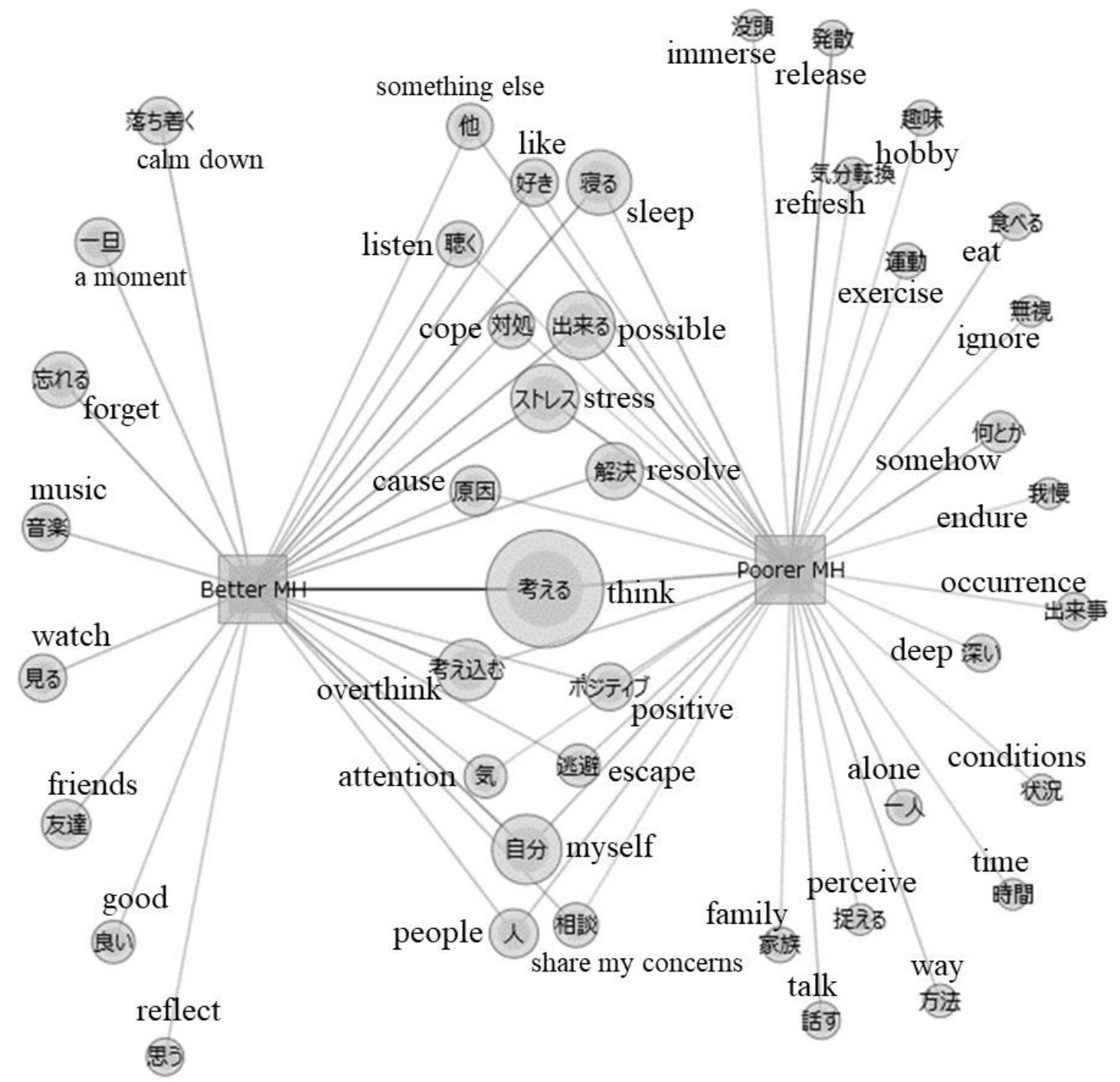

Figure 1. Co-occurrence network in the low-SPS group

Notes. Better $\mathrm{MH}=$ better mental health subgroup, Poorer $\mathrm{MH}=$ poorer mental health subgroup.

The squares represent grouping variables (i.e., subgroups created based on mental health level). The circles refer to components that co-occur with one or two grouping variable(s). 


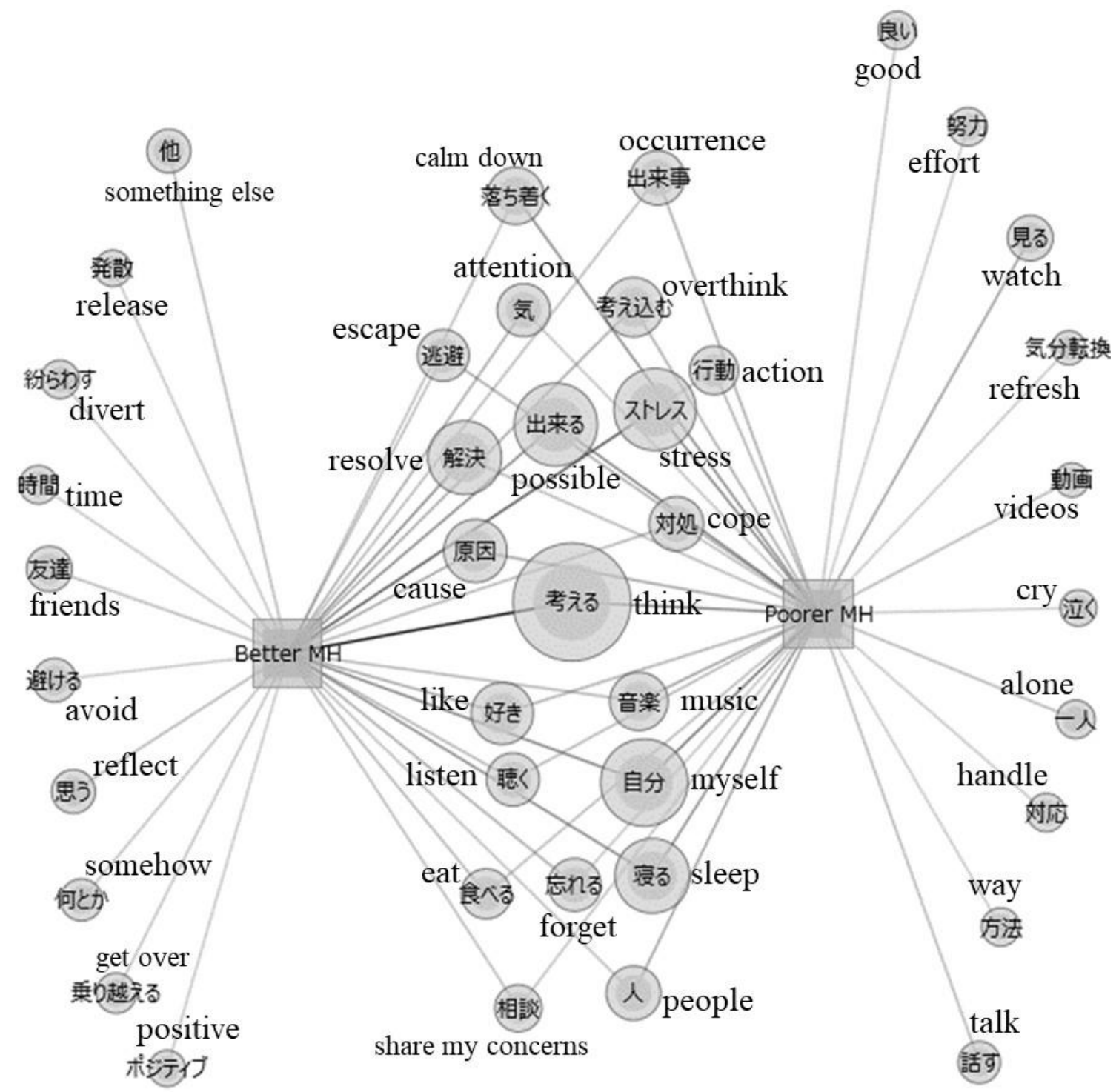

Figure 2. Co-occurrence network in the medium-SPS group

Notes. Better $\mathrm{MH}=$ better mental health subgroup, Poorer $\mathrm{MH}=$ poorer mental health subgroup.

The squares represent grouping variables (i.e., subgroups created based on mental health level). The circles refer to components which co-occur with one or two grouping variable(s). 


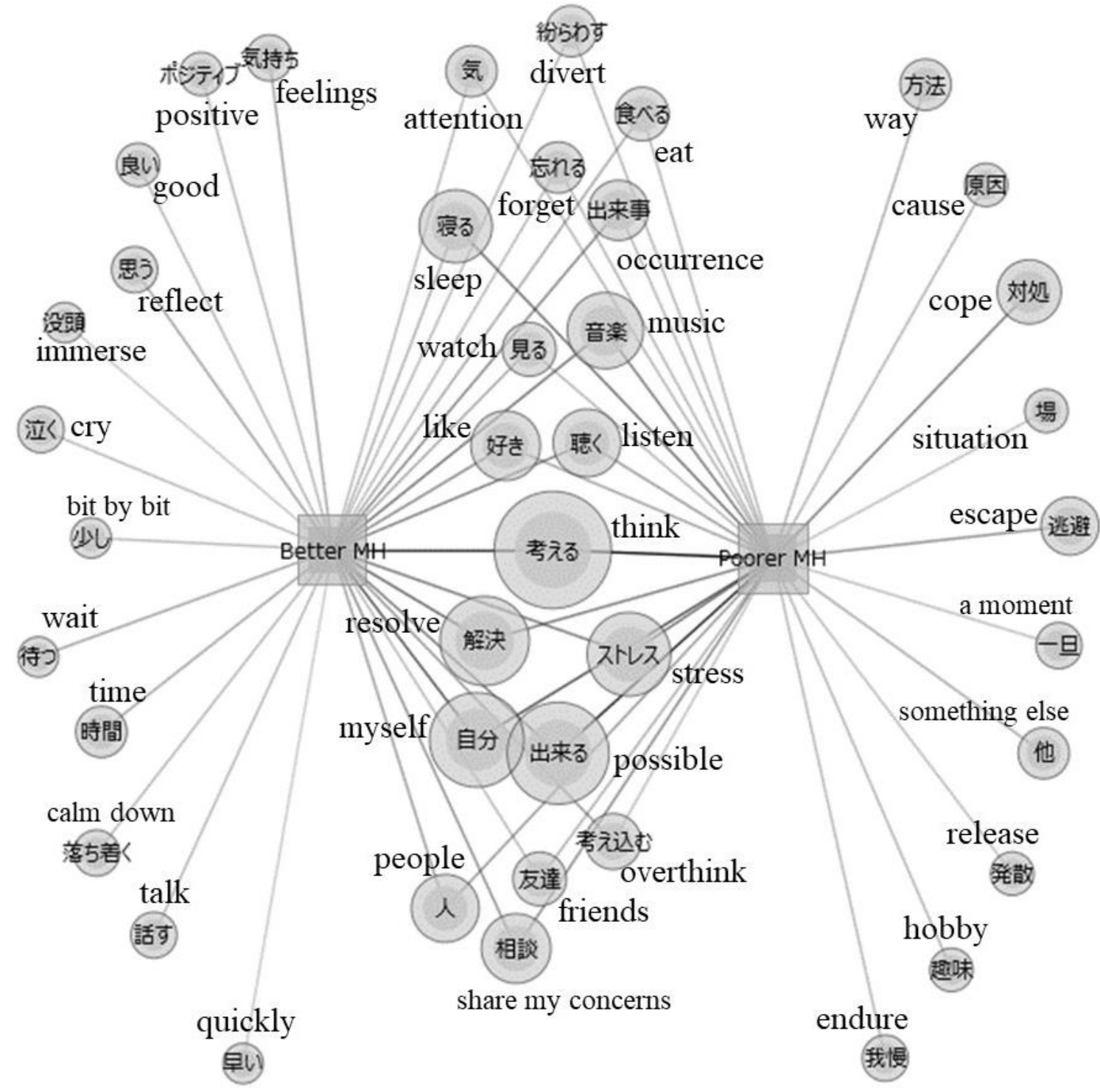

Figure 3. Co-occurrence network in the high-SPS group

Notes. Better $\mathrm{MH}=$ better mental health subgroup, Poorer $\mathrm{MH}=$ poorer mental health subgroup.

The squares represent grouping variables (i.e., subgroups created based on mental health level). The circles refer to components which co-occur with one or two grouping variable(s). 